\title{
Trends in the properties and structures of the simple metals from a universal local pseudopotential
}

\author{
Fernando Nogueira and Carlos Fiolhais \\ Department of Physics, University of Coimbra, P3000 Coimbra, Portugal \\ John P. Perdew \\ Department of Physics and Quantum Theory Group, Tulane University, New Orleans, Louisiana
}

(Received 4 August 1998)

\begin{abstract}
The properties of simple metals are fixed primarily by the equilibrium average valence-electron density parameter $r_{s}$, and secondarily by the valence $z$. The simplest level of theory that can account quantitatively for these trends invokes a "universal", local electron-ion pseudopotential, defined for each pair $\left(r_{s}, z\right)$ and treated as a second-order perturbation. We construct this pseudopotential from two conditions: (1) The total energy should minimize at the equilibrium Wigner-Seitz radius $z^{1 / 3} r_{s}$. (2) The bulk modulus should equal the realistic $r_{s}$-dependent prediction of the stabilized jellium model with effective valence $z^{*}=1$. These conditions can be satisfied by an analytic local pseudopotential depending upon two parameters other than $z$; we show that the choice of the two-parameter form (evanescent core vs Heine-Abarenkov) is not important. Our universal local pseudopotential is applied to calculate realistic bulk binding energies, pressure derivatives of bulk moduli, Voigt shear moduli, and interstitial electron numbers, revealing their trends as functions of $r_{s}$ and $z$. Equilibrium crystal structures are mapped in the $r_{s}-z$ plane, where the Hume-Rothery rules for substitutional alloys are manifest. The effect of pressure on crystal structure is also examined. [S0163-1829(99)10603-9]
\end{abstract}

\section{INTRODUCTION}

The 16 simple or $s p$-bonded metals with valence $z \leqslant 4$ (Be, Al, Ga, Sn, Pb, In, Tl, Mg, Li, Ca, Sr, Ba, Na, K, Rb, and $\mathrm{Cs}$ ) display a weak effective interaction between valence electrons and ion cores. ${ }^{1,2}$ In previous work, ${ }^{3}$ we constructed local electron-ion pseudopotentials for each metal individually. In conjunction with the local-density approximation for exchange and correlation, these pseudopotentials successfully predict the bulk binding energies, bulk moduli, and phonon frequencies, ${ }^{4,5}$ and structural energy differences for most of these metals in the face-centered-cubic (fcc), ideal hexagonal-close-packed (hcp), and body-centered-cubic (bcc) structures, as well as the liquid-metal resistivities. ${ }^{4}$ The transferability of these pseudopotentials beyond the condensed state varies strongly from one element to another. ${ }^{6}$

The simple metals are amenable to theoretical description at many different levels. The lowest level is the jellium model, in which the valence electrons of density

$$
\bar{n}=\frac{3}{4 \pi r_{s}^{3}}
$$

neutralize a uniform positive background representing the ion lattice. Jellium is a fairly realistic model for $r_{s}$ $\simeq 4$ bohr $(\mathrm{Na})$, where it is stable, but not for $r_{s} \simeq 2(\mathrm{Al})$, where its surface energy is negative, or for $r_{s} \sim 6(\mathrm{Cs})$, where its bulk modulus is negative. The stabilized jellium model ${ }^{7,8}$ with effective valence $z^{*}=1$ remedies these ills without losing the simplicity of the jellium model, and even predicts realistic bulk moduli for the simple metals.

The jellium model and the stabilized jellium model with $z^{*}=1$ have a single input parameter $r_{s}$. Another such model is the ideal metal of Shore and Rose, ${ }^{9}$ which can be viewed ${ }^{10}$ as the $z^{*} \rightarrow 0$ limit of stabilized jellium. However, $z^{*}=1$ is a more realistic choice which is not only correct for the alkali metals but also more generally consistent with neglect of the band structure energy or of variations in the valence-electron density.

While $r_{s}$ is the dominant density parameter of the simple metals, the valence $z$ plays a secondary role and has a significant effect on the bulk binding energy and the pressure derivative of the bulk modulus. The stabilized jellium model with the true valence $z$, also known as the structureless pseudopotential model, ${ }^{7}$ correctly describes the dependence of the bulk binding energy upon $r_{s}$ and $z$, but spoils some of the bulk moduli for the polyvalent simple metals that were correctly described by the choice $z^{*}=1$. The stabilized jellium model is derived from the local pseudopotential picture by constraining the valence-electron density to be uniform and then choosing the average value of the core repulsion to make the total energy minimize at the observed Wigner-Seitz radius $z^{1 / 3} r_{s}$. The constraint of uniform density is fairly realistic for $z \leqslant 2$, but not for $z>2$.

The appropriate level of theory to describe the broad trends of the simple metals is one which invokes a "universal" local electron-ion pseudopotential, defined for each pair $\left(r_{s}, z\right)$ and treated as a second-order perturbation on the energy of the uniform electron gas. ${ }^{1,1-14}$ This is also the lowest level of theory that can predict shear moduli and crystal structures. Universal local pseudopotentials were sought by Ling and Gelatt ${ }^{13}$ to explain the "chemical trends" of the simple metals, and in our earlier work, ${ }^{3}$ but without great success. (Although Ling and Gelatt found some chemical trends of the elastic constants, their universal local pseudopotential was sometimes inconsistent with Fermi-surface properties and liquid-metal resistivities.) Here we will fill in this missing link in the hierarchy of theoretical models, be- 


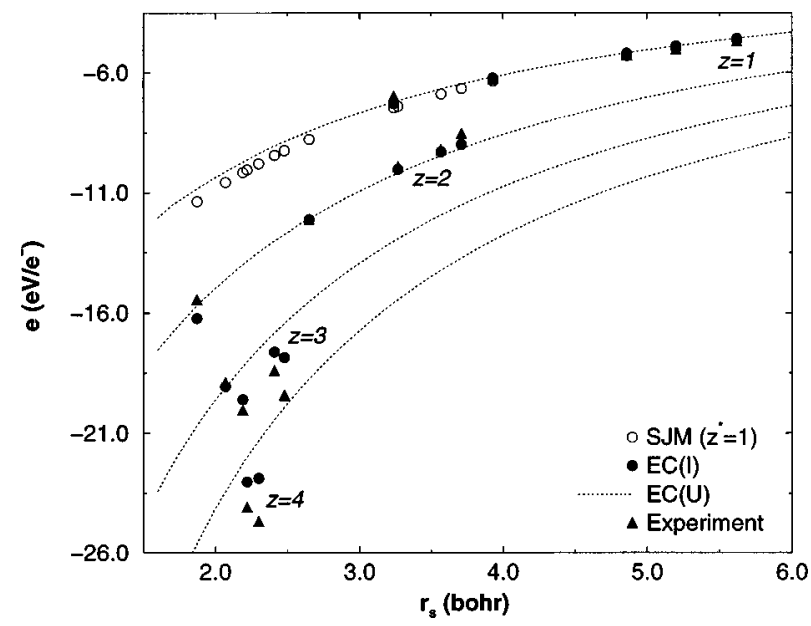

FIG. 1. Bulk binding energies calculated with the stabilized jellium model with $z^{*}=1\left[\operatorname{SJM}\left(z^{*}=1\right)\right]$ and the individual evanescent core pseudopotential $[\mathrm{EC}(\mathrm{I})]$ in second-order perturbation theory, compared to one another and to experimental results (as in Ref. 3). The dashed lines represent the universal EC(U) values of Sec. III. $r_{s}$ and $z$ are the equilibrium density parameter and the valence, respectively. To identify the individual metals, we refer to Table I. (The bulk binding energy is the energy per valence electron needed to break up the solid into separated valence electrons and ions.)

tween the stabilized jellium level and the level of "individual" pseudopotentials constructed separately for each element. At the latter level, Hafner and Heine ${ }^{15,16}$ related crystal structures to features of the screened pseudopotential.

Our recently constructed individual local pseudopotentials ${ }^{3}$ are analytic " evanescent-core" functions in real space, depending upon two parameters other than the valence $z$. The two parameters were fixed by two conditions: (1) The total energy per electron, evaluated to second order in the pseudopotential, should minimize at the observed Wigner-Seitz radius $z^{1 / 3} r_{s}$. (2) The interstitial electron number $N_{\text {int }}$ (i.e., the number of electrons between the surface of the Wigner-Seitz cell and an inscribed sphere), evaluated to first order in the pseudopotential, should match that found in an all-electron calculation.

To construct universal local pseudopotentials, here we shall replace condition (2) by a condition which depends upon $r_{s}$ alone and gives similar results: $\left(2^{\prime}\right)$ The bulk modulus should equal the realistic prediction of the stabilized jellium model with effective valence $z^{*}=1$, an analytical function of $r_{s}$. The absence of a second condition in the work of Ling and Gelatt, ${ }^{13}$ and use of an unrealistic second condition in our earlier attempt to construct the universal pseudopotential, ${ }^{3}$ were responsible for the lack of success encountered there.

Figures 1-5 display the trends of the simple-metal bulk binding energy $e$, bulk modulus $B$, pressure derivative $B^{\prime}$ of the bulk modulus, Voigt shear modulus $\mu$, and interstitial electron number $N_{\text {int }}$, as functions of $r_{s}$ and $z$. These figures show not only the experimental values, but also the predictions of the stabilized jellium model (SJM) with effective valence $z^{*}=1$, the individual evanescent core pseudopotential [EC(I), using the conventional crystal structures of Ref. $3]$, and the universal evanescent core pseudopotential [EC (U), using the fcc structure]. Like the equilibrium $r_{s}$,

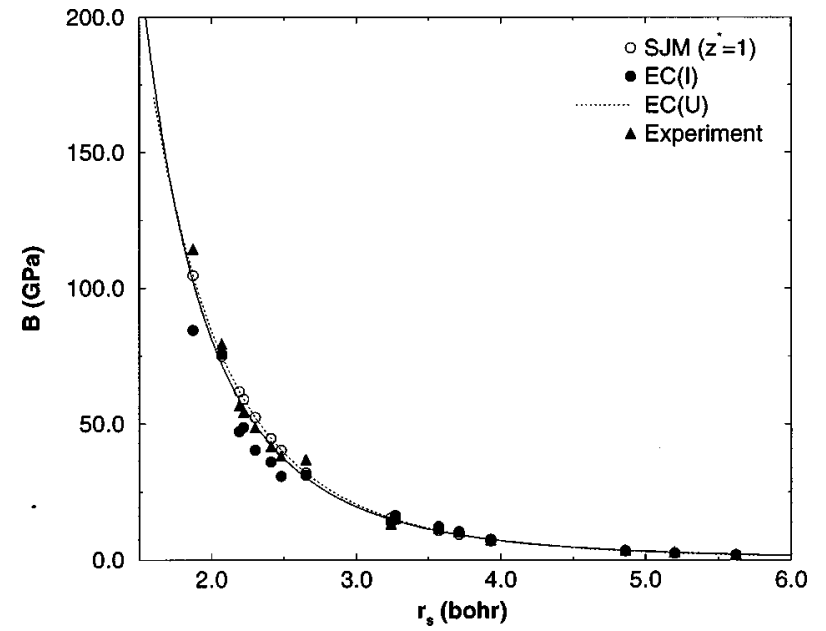

FIG. 2. Same as Fig. 1 for the bulk moduli. The EC(U) results coincide with the $\operatorname{SJM}\left(z^{*}=1\right)$ values by construction. The solid line is the curve obtained by fitting $A r_{s}^{-7 / 2}$ to the $\operatorname{SJM}\left(z^{*}=1\right)$ points. $A$ is $918 \mathrm{GPa}$ bohr $^{7 / 2}$. Experimental values are as in Refs. 3 and 17.

the properties $e, B$, and $\mu$ vary little among the fcc, hcp, and bcc structures. ${ }^{4,13}$ The EC(I) and experimental values are those in the corrected version of Ref. 3, although a few of the experimental values have been updated. ${ }^{17}$ Our universal model postulates positive bulk moduli $B$, indicating stability under expansion and compression, and finds positive Voigt shear moduli $\mu$. Note that $r_{\mathrm{WS}}=z^{1 / 3} r_{s}, B$, and $B^{\prime}$ are themselves inputs to a "universal" equation of state, ${ }^{10,18}$ which predicts the pressure $P$ for any volume compression ratio $V / V_{0}$

While the bulk modulus of Fig. 2 (like the surface properties discussed in Refs. 7, 8, and 19) is determined largely by $r_{s}$ alone, the other properties clearly depend upon $z$ as well. The universal pseudopotential results, which are defined for all pairs $\left(r_{s}, z\right)$, reveal trends that would otherwise be hard to see due to the sparseness of the real simple metals in the $r_{s}-z$ plane and to individual variations among the elements.

In Sec. II, after recalling some of the basics of pseudopo-

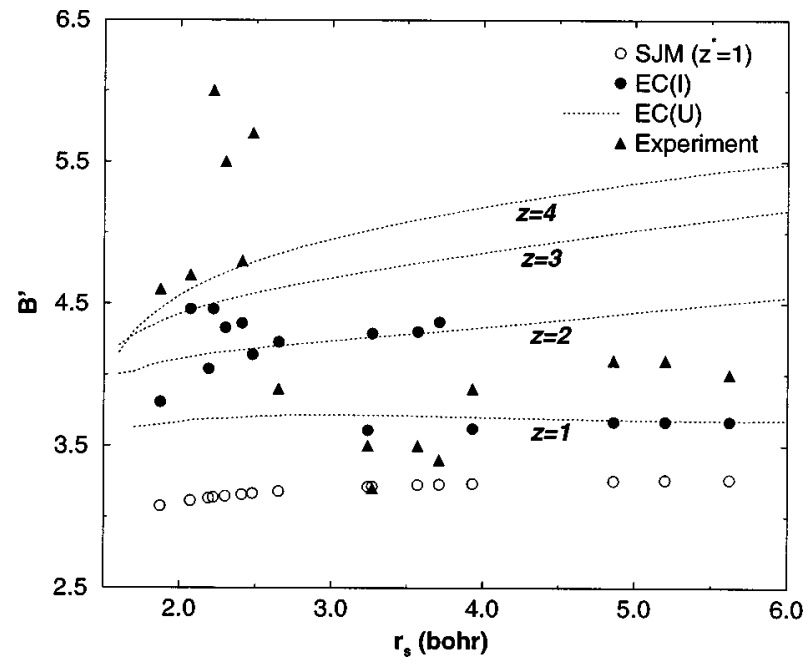

FIG. 3. Same as Fig. 1 for the pressure derivatives of the bulk moduli. 


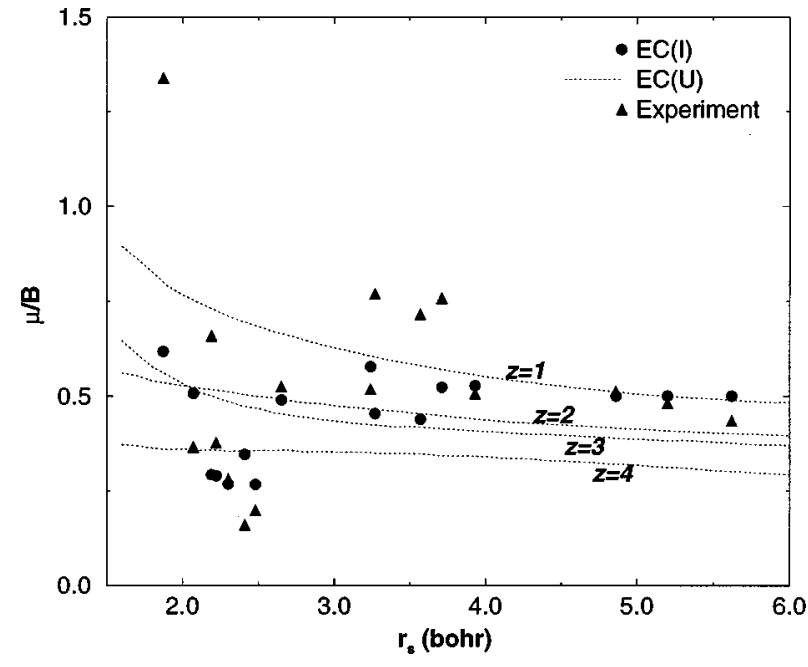

FIG. 4. Same as Fig. 1 for the Voigt (spherically averaged) shear moduli $\mu$. Note that all shear moduli vanish in the stabilized jellium model. Experimental values are as in Ref. 4.

tential perturbation theory, we shall compare two different two-parameter local pseudopotentials (evanescent core and Heine-Abarenkov) fitted to the same individual conditions (1) and (2) above. Although these two pseudopotentials look rather different, we find that they predict essentially the same physical properties for the simple metals.

In Sec. III, we will discuss condition (2') for the construction of our universal local pseudopotential. We will also find universal phase diagrams for the equilibrium crystal structures of pure metals and alloys under zero and high pressures, which show that the valence $z$ largely controls the structure. While the pure metals are sparse in the $\left(r_{s}, z\right)$ plane, the alloys cover far more of this plane and represent a rich field of potential application for our work. Our conclusions and ideas for future work are summarized in Sec. IV.

\section{PERTURBATION THEORY AND PSEUDOPOTENTIAL FORMS}

Local pseudopotentials are easy to use and economical for simultaneous treatment of many metals and structures, lead-

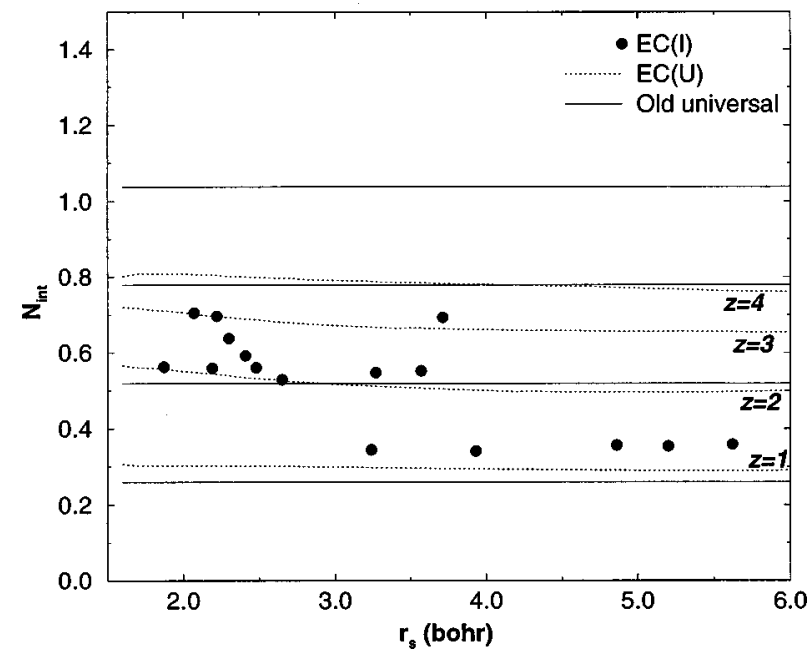

FIG. 5. Number of interstitial electrons as a function of the density parameter $r_{s}$ and the valence. ing to identification of trends along the periodic table and to physical insight. Furthermore, density functional theory requires in principle that the external potential be local. ${ }^{20}$

We may take as a perturbation on jellium (or better, on stabilized jellium with $z^{*}=1$ ) the difference between the local pseudopotential and the jellium potential. Within second-order perturbation theory, ${ }^{1,11-14}$ the binding energy per valence electron is

$$
e=e_{J}+e_{M}+\bar{w}_{R}+e_{\mathrm{BS}}
$$

where $e_{J}$ is the jellium energy (which includes only kinetic and exchange-correlation contributions) and $e_{M}$ is the Madelung or Ewald energy, which describes the electrostatic interaction between ions in a lattice. $\bar{w}_{R}$ is the average in the Wigner-Seitz cell of the repulsive part of the pseudopotential, and $e_{\mathrm{BS}}$ is the second-order contribution or bandstructure energy:

$$
\begin{gathered}
\bar{w}_{R}=\frac{\bar{n}}{z} \int_{0}^{\infty}\left[w(r)+\frac{z}{r}\right] 4 \pi r^{2} d r, \\
e_{\mathrm{BS}}=\frac{\bar{n}}{2 z^{2}} \sum_{\vec{G} \neq 0}|w(G) S(\vec{G})|^{2} \frac{\chi(G)}{\epsilon(G)} .
\end{gathered}
$$

Here $w(r)$ and $w(G)$ are, respectively, the pseudopotential and its Fourier transform, $\vec{G}$ is a reciprocal-lattice vector, $S(\vec{G})$ is the structure factor, $\chi(G)$ is the susceptibility, and $\epsilon(G)$ is the dielectric function, as defined in Ref. 3. The lattice-dependent terms in Eq. (2) are $e_{M}$ and $e_{\mathrm{BS}}$.

The stabilized jellium model ${ }^{7}$ drops $e_{\mathrm{BS}}$ in Eq. (2), and makes a spherical approximation for $e_{M}$. Thus its energy is independent of the lattice structure. The spherical approximation for $e_{M}$ introduces negligible errors for the fcc, hcp, and bec lattices.

The local Heine-Abarenkov (HA) (Refs. 12 and 21-23) or Cohen ${ }^{24}$ potential can be regarded as a generalization of the Ashcroft empty-core potential. ${ }^{25}$ The HA potential is constant inside the core but not necessarily zero. It may be written in real space as

$$
w^{\mathrm{HA}}\left(r ; r_{c}, u\right)=\left\{\begin{array}{cc}
u z / r_{c}, & r \leqslant r_{c} \\
-z / r, & r>r_{c} .
\end{array}\right.
$$

For $u=0$, Eq. (5) reduces to the Ashcroft potential, and, for $u=-1$, to a continuous potential with a discontinuous first derivative, the one-parameter Shaw potential ${ }^{26}$ used by Ling and Gelatt ${ }^{13}$ in their search for a universal pseudopotential. In order to determine the two parameters of the HA potential, different information has been invoked: solid state (band structures, Fermi-surface data, bulk moduli, etc.) or atomic (excitation energies, scattering data, etc.). ${ }^{27-36}$ Some authors ${ }^{27-34}$ fit the parameters to the equilibrium lattice constant and measured bulk modulus (or bulk phonon frequencies).

In Ref. 3, we introduced a potential which also depends on two parameters but is smoother than the HA potential. This evanescent core (EC) pseudopotential has the general form 

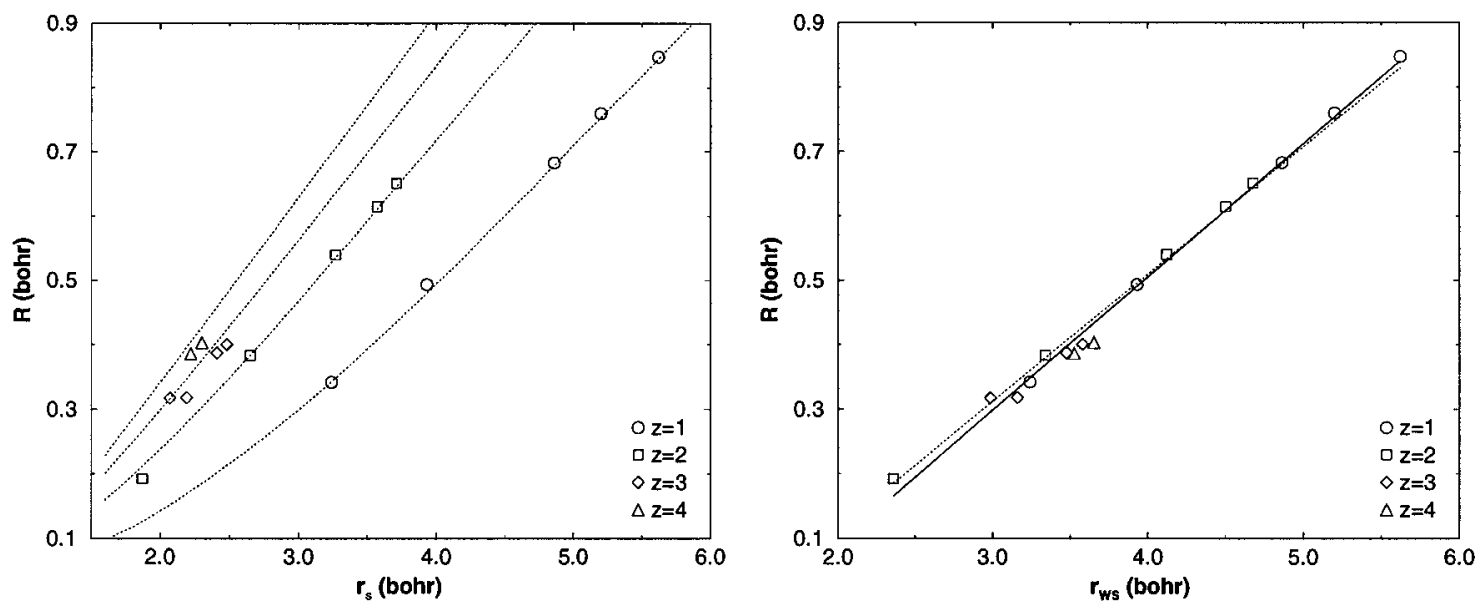

FIG. 6. Core decay length $R$ of the EC(I) pseudopotential as a function of the density parameter $r_{s}$ and of the Wigner-Seitz radius $r_{\mathrm{WS}}=z^{1 / 3} r_{s}$. Left: the dashed lines show the EC(U) parameters of Sec. III, while the symbols denote the EC(I) parameters. Right: the full line is a linear rms fit to the EC(I) points. The dashed line is a linear rms fit to the EC(U) parameters of the simple metals. Note that $R$ is small for metastable metallic hydrogen $\left(z=1, r_{s}=1.7\right)$.

$$
w^{\mathrm{EC}}(r ; R, \alpha)=-\frac{z}{r}+w_{R}(r ; R, \alpha)
$$

and is constructed so that the repulsive part $w_{R}(r ; R, \alpha)$ shows an exponential decay at large $r$, with $R$ the decay length. In the opposite limit of small $r$, some analytical conditions at the origin assure smoothness (a property which is emphasized, for instance, in the Troullier-Martins construction of pseudopotentials $\left.{ }^{37,38}\right)$. Its Fourier transform, which may be found analytically, has a single zero controlled by $R$ and $\alpha$. The parameters of this potential are chosen using a crystalline reference state or conventional lattice, in contrast to the usual construction of the nonlocal norm-conserving pseudopotential, which starts from the free atom. ${ }^{37-42} \mathrm{We}$ have chosen the observed room-temperature lattice except for $\mathrm{Ga}, \mathrm{In}$, and $\mathrm{Sn}$, which for simplicity were taken to be fcc. The room-temperature lattice is also the zero-temperature lattice, except for $\mathrm{Li}$ and $\mathrm{Na},{ }^{43-45}$ where the former is bcc instead.

The potential parameters were then adjusted to reproduce the key features of the valence electron density. Besides the stability condition

$$
\frac{\partial}{\partial r_{s}} e\left(r_{s}, z ; R, \alpha\right)=0,
$$

which assures that the total energy minimizes at the experimental electron density, we used the condition

$$
N_{\text {int }}=N_{\text {int }}^{\text {all-electron }},
$$

which assures that the interstitial electron number $N_{\text {int }}$ obtained in an all-electron calculation is reproduced within first-order perturbation theory (Fig. 5). The EC potential with the parameters thus determined for each individual metal is referred to as EC(I).

In Figs. 6 and 7 we present the parameters $R$ and $\alpha$ which were found with our approach as a function of $r_{s}$ for each $z$. $R$ shows a good linear correlation with the Wigner-Seitz radius $r_{\mathrm{WS}}=z^{1 / 3} r_{s}$ for any $z . \alpha$ is essentially a smooth function of $r_{s}$ for each $z$.
We have also used the conditions given by Eqs. (7) and (8) to extract the parameters of the HA potential. The HA potential thus found is denoted HA(I). Table I shows the resulting parameters, which also display trends as functions of $r_{s}$ and $z$. The rms errors in the calculated binding energies and bulk moduli with respect to experiment are only slightly larger with the HA(I) potential than with the EC(I) $(5 \%$ and $17 \%$, respectively, in contrast to $4 \%$ and $13 \%$ ). The form of a two-parameter local pseudopotential is therefore less important than the choice of properties to be fitted by those two parameters.

In Table II, we present the predicted crystal structures for those simple metals which are experimentally cubic or hcp, comparing the EC(I) with the HA(I) potentials. EC(I) correctly predicts nine structures out of 13 , while $\mathrm{HA}(\mathrm{I})$ predicts seven. The failure of EC(I) occurs only for $\mathrm{Ca}, \mathrm{Sr}, \mathrm{Ba}$, and $\mathrm{Pb}$. Table III presents energy differences for three representative simple metals, corresponding to the valences 1,2 , and 3. The local pseudopotential perturbative approaches EC(I) and HA(I) do very well in comparison with other, more sophisticated, approaches. ${ }^{46-48}$

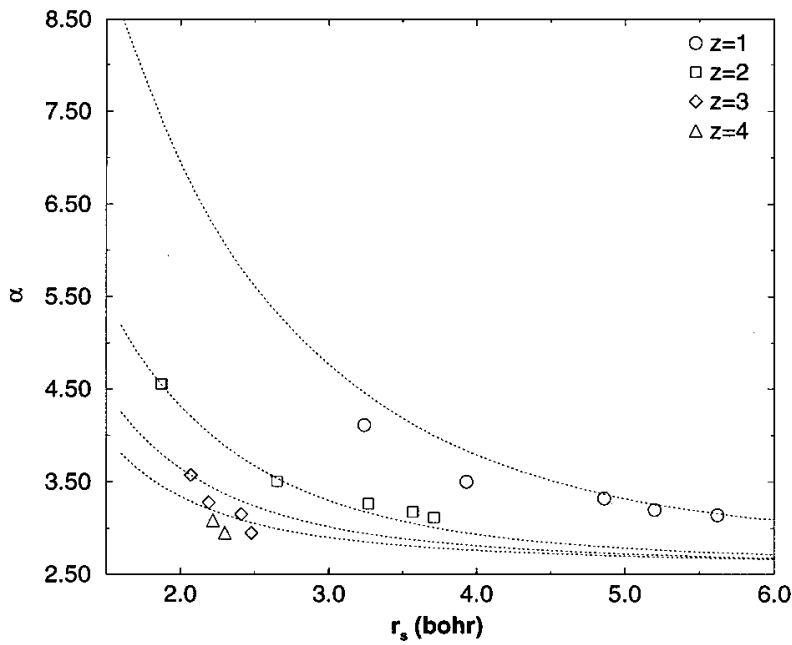

FIG. 7. Pseudopotential parameter $\alpha$ plotted against the density parameter $r_{s}$. Symbols and lines are as in Fig. 6, left. 
TABLE I. Density parameter $r_{s}$, valence $z$, and individual Heine-Abarenkov [HA(I)] pseudopotential parameters for the simple metals, and the corresponding binding energies, bulk moduli, and pressure derivatives of the bulk moduli. Lengths are given in bohr, energies in eV per electron, and bulk moduli in GPa. The values in parentheses are the experimental values, as summarized in Refs. 3 and 17.

\begin{tabular}{|c|c|c|c|c|c|c|c|}
\hline Metal & $r_{s}$ & $z$ & $r_{c}$ & $u$ & $e$ & $B$ & $B^{\prime}$ \\
\hline $\mathrm{Be}$ & 1.87 & 2 & 0.779 & 0.416 & $\begin{array}{c}-16.21 \\
(-15.45)\end{array}$ & $\begin{array}{c}77.4 \\
(114.4)\end{array}$ & $\begin{array}{c}3.8 \\
(4.6)\end{array}$ \\
\hline $\mathrm{Al}$ & 2.07 & 3 & 1.377 & -0.453 & $\begin{array}{c}-19.07 \\
(-18.88)\end{array}$ & $\begin{array}{c}67.1 \\
(79.4)\end{array}$ & $\begin{array}{c}4.7 \\
(4.7)\end{array}$ \\
\hline $\mathrm{Ga}$ & 2.19 & 3 & 1.481 & -0.705 & $\begin{array}{c}-19.64 \\
(-20.03)\end{array}$ & $\begin{array}{c}42.6 \\
(56.8)\end{array}$ & 4.1 \\
\hline Sn & 2.22 & 4 & 1.951 & -0.898 & $\begin{array}{c}-23.15 \\
(-24.08)\end{array}$ & $\begin{array}{c}41.6 \\
(54.1)\end{array}$ & $\begin{array}{c}4.6 \\
(6.0)\end{array}$ \\
\hline $\mathrm{Pb}$ & 2.30 & 4 & 2.350 & -1.030 & $\begin{array}{c}-22.17 \\
(-24.68)\end{array}$ & $\begin{array}{c}38.6 \\
(48.8)\end{array}$ & $\begin{array}{c}4.8 \\
(5.5)\end{array}$ \\
\hline In & 2.41 & 3 & 1.963 & -0.876 & $\begin{array}{c}-17.70 \\
(-18.40)\end{array}$ & $\begin{array}{c}33.7 \\
(41.8)\end{array}$ & $\begin{array}{c}4.5 \\
(4.8)\end{array}$ \\
\hline $\mathrm{Tl}$ & 2.48 & 3 & 2.256 & -0.993 & $\begin{array}{c}-17.30 \\
(-19.42)\end{array}$ & $\begin{array}{c}30.9 \\
(38.2)\end{array}$ & $\begin{array}{c}4.5 \\
(5.7)\end{array}$ \\
\hline $\mathrm{Mg}$ & 2.65 & 2 & 1.741 & -0.571 & $\begin{array}{c}-12.15 \\
(-12.11)\end{array}$ & $\begin{array}{c}30.3 \\
(36.9)\end{array}$ & $\begin{array}{c}4.4 \\
(3.9)\end{array}$ \\
\hline $\mathrm{Li}$ & 3.24 & 1 & 1.482 & -0.127 & $\begin{array}{c}-7.27 \\
(-6.97)\end{array}$ & $\begin{array}{c}13.8 \\
(13.3)\end{array}$ & $\begin{array}{c}3.7 \\
(3.5)\end{array}$ \\
\hline $\mathrm{Ca}$ & 3.27 & 2 & 2.599 & -0.774 & $\begin{array}{l}-10.08 \\
(-9.91)\end{array}$ & $\begin{array}{c}16.9 \\
(15.2)\end{array}$ & $\begin{array}{c}4.5 \\
(3.2)\end{array}$ \\
\hline $\mathrm{Sr}$ & 3.57 & 2 & 3.071 & -0.858 & $\begin{array}{c}-9.35 \\
(-9.21)\end{array}$ & $\begin{array}{c}12.8 \\
(11.6)\end{array}$ & $\begin{array}{c}4.5 \\
(3.5)\end{array}$ \\
\hline $\mathrm{Ba}$ & 3.71 & 2 & 3.425 & -0.936 & $\begin{array}{c}-9.03 \\
(-8.54)\end{array}$ & $\begin{array}{c}11.1 \\
(10.3)\end{array}$ & $\begin{array}{c}4.6 \\
(3.4)\end{array}$ \\
\hline $\mathrm{Na}$ & 3.93 & 1 & 2.371 & -0.660 & $\begin{array}{c}-6.24 \\
(-6.25)\end{array}$ & $\begin{array}{c}7.1 \\
(7.3)\end{array}$ & $\begin{array}{r}3.7 \\
(3.9)\end{array}$ \\
\hline $\mathrm{K}$ & 4.86 & 1 & 3.362 & -0.774 & $\begin{array}{c}-5.19 \\
(-5.27)\end{array}$ & $\begin{array}{c}3.5 \\
(3.7)\end{array}$ & $\begin{array}{c}3.8 \\
(4.1)\end{array}$ \\
\hline $\mathrm{Rb}$ & 5.20 & 1 & 3.897 & -0.870 & $\begin{array}{c}-4.91 \\
(-5.02)\end{array}$ & $\begin{array}{c}2.8 \\
(2.9)\end{array}$ & $\begin{array}{c}3.7 \\
(4.1)\end{array}$ \\
\hline Cs & 5.62 & 1 & 4.486 & -0.926 & $\begin{array}{c}-4.59 \\
(-4.68)\end{array}$ & $\begin{array}{c}2.1 \\
(2.3)\end{array}$ & $\begin{array}{r}3.7 \\
(4.0)\end{array}$ \\
\hline
\end{tabular}

\section{UNIVERSALITY CONDITIONS AND UNIVERSAL PHASE DIAGRAMS}

From Figs. 6 and 7, it is apparent that the pseudopotential parameters are simple functions of $r_{s}$ and $z$. By a universal pseudopotential, we mean one whose parameters are only determined by the equilibrium density $r_{s}$ and the valence $z$. In Ref. 3 we presented a universal pseudopotential which required two conditions: the stability condition and the equality of the interstitial average density to the bulk average density. As shown in Fig. 5, the second condition was realistic for $z \leqslant 2$, but not for $z=3$ and 4. Pseudopotentials for high-valence metals draw electrons out of the interstitial region. This effect reduces $B$ from the too-high values predicted by the stabilized jellium model with the true valence $z$. Here we propose a more accurate universal pseudopotential model $[\mathrm{EC}(\mathrm{U})]$. We could as well use the HA potential form
TABLE II. Equilibrium structural phases as predicted by several potentials: individual evanescent core potential [EC(I)], individual Heine-Abarenkov potential [HA(I)], and universal evanescent core potential $[\mathrm{EC}(\mathrm{U})]$. The last column lists the observed zerotemperature structures. (We assumed hcp to be the zero-temperature structure for $\mathrm{Li}$ and $\mathrm{Na}$, since it is similar to the 9R lattice). The symbol $\sqrt{ }$ means that the observed structure is reproduced.

\begin{tabular}{|c|c|c|c|c|}
\hline Metal & $\mathrm{EC}(\mathrm{I})$ & $\mathrm{HA}(\mathrm{I})$ & $\mathrm{EC}(\mathrm{U})$ & Expt. \\
\hline $\mathrm{Be}$ & $\sqrt{ }$ & $\sqrt{ }$ & $\sqrt{ }$ & hcp \\
\hline $\mathrm{Al}$ & $\sqrt{ }$ & $\sqrt{ }$ & $\sqrt{ }$ & fcc \\
\hline $\mathrm{Pb}$ & hcp & hcp & hcp & fcc \\
\hline $\mathrm{Tl}$ & $\sqrt{ }$ & fcc & fcc & hcp \\
\hline $\mathrm{Mg}$ & $\sqrt{ }$ & $\sqrt{ }$ & $\sqrt{ }$ & hcp \\
\hline $\mathrm{Li}$ & $\sqrt{ }$ & fcc & bcc & hcp \\
\hline $\mathrm{Ca}$ & hcp & hcp & hcp & fcc \\
\hline $\mathrm{Sr}$ & hcp & hcp & hcp & fcc \\
\hline $\mathrm{Ba}$ & hcp & hcp & hcp & bcc \\
\hline $\mathrm{Na}$ & $\sqrt{ }$ & $\sqrt{ }$ & bcc & hcp \\
\hline $\mathrm{K}$ & $\sqrt{ }$ & $\sqrt{ }$ & $\sqrt{ }$ & bcc \\
\hline $\mathrm{Rb}$ & $\sqrt{ }$ & $\sqrt{ }$ & $\sqrt{ }$ & bcc \\
\hline Cs & $\sqrt{ }$ & $\sqrt{ }$ & $\sqrt{ }$ & bcc \\
\hline
\end{tabular}

with similar results, but we prefer the smooth EC model, which leads to a more rapid convergence of sums over reciprocal lattice vectors.

The EC(U) pseudopotential parameters $R$ and $\alpha$ are found from $r_{s}$ and $z$ with the help of two conditions: (1) The energy per electron should minimize at the observed equilibrium Wigner-Seitz radius $z^{1 / 3} r_{s}$. (2') The calculated bulk modulus $B$ should equal the realistic prediction of the stabilized jellium model with effective valence $z^{*}=1$, an analytic function of $r_{s}$ which can be represented simply by the approximation $B=A r_{s}^{-7 / 2}$ (as shown in Fig. 2). Cohen ${ }^{49}$ and Kelires $^{50}$ found that a similar form with the same exponent describes the bulk moduli of semiconductors. The individual-pseudopotential analog of condition $\left(2^{\prime}\right)$ would fit to the observed bulk modulus for each metal, as some users of the Heine-Abarenkov form have done. ${ }^{27-34}$

While condition (1) follows from first principles, condition $\left(2^{\prime}\right)$ is ultimately supported by comparison with experiment. However, results only slightly less satisfactory are obtained if we replace condition $\left(2^{\prime}\right)$ by minimization of the core decay length $R$, which helps to maximize transferability of the pseudopotential.

TABLE III. Structural energy differences for some representative metals, as predicted by the potentials indicated in Table II. Also shown are results from perturbative (GPT, Ref. 46) and nonperturbative calculations (NP, Refs. 47 and 48). All energies are in $\mathrm{meV}$ per electron.

\begin{tabular}{rrrrrrc}
\hline \hline \multicolumn{2}{c}{ Metal } & EC(I) & HA(I) & EC(U) & GPT & NP \\
\hline \multirow{2}{*}{$\mathrm{Na}$} & fcc-bcc & -0.4 & -0.6 & 0.3 & -0.7 & 1.4 \\
& fcc-hcp & 0.1 & 0.1 & -0.1 & 0.1 & 3.5 \\
$\mathrm{Mg}$ & fcc-bcc & -9.5 & -10.3 & -8.9 & -9.5 & - \\
& fcc-hcp & 4.1 & 3.6 & 5.1 & 4.1 & - \\
$\mathrm{Al}$ & fcc-bcc & -29.4 & -32.3 & -28.5 & -33.1 & -38.2 \\
& fcc-hcp & -7.7 & -8.4 & -7.3 & -7.7 & -16.6 \\
\hline \hline
\end{tabular}


From Eq. (2.15) of Ref. 3, we can compute $q_{0}$, the first and only zero of the Fourier transform $w(q)$ of the universal pseudopotential, and its ratio to $2 k_{F}$, where $k_{F}$ $=(9 \pi / 4)^{1 / 3} / r_{s}$ is the bulk Fermi wave vector. For $z=1$, $q_{0} / 2 k_{F}$ varies from 0.71 at $r_{s}=1.6$ to 0.93 at $r_{s}=6$. The range is even narrower at $z=2$ (0.75 to 0.87$), z=3$ (0.76 to $0.78)$, and $z=4$ (0.76 to 0.73 ).

The EC(I) and EC(U) pseudopotential parameters are compared in Figs. 6 and 7, and some physical results are compared in Figs. 1-5 and Tables II and III. The predicted phases in Table II are the same, except for $\mathrm{Li}, \mathrm{Na}$, and $\mathrm{Tl}$. [For $\mathrm{Li}$ and $\mathrm{Na}, \mathrm{EC}(\mathrm{U})$ predicts the conventional bcc phase.] The structural energy differences are also very close for the three metals considered. The rms errors in the $\mathrm{EC}(\mathrm{U})$ binding energies and bulk moduli (Figs. 1 and 2 ) are $7 \%$ and $8 \%$, i.e., the bulk modulus error has been reduced at the expense of the binding energy error.

Figure 1 shows how the universal pseudopotential clarifies the systematics of the binding energies. For fixed $z$, the energy decreases with decreasing $r_{s}$, and, for a given $r_{s}$, the energy increases with decreasing $z$. Figure 3 shows that, for a given $z$, the pressure derivative $B^{\prime}$ of the bulk modulus increases slowly with $r_{s}$ and that, for each $r_{s}, B^{\prime}$ increases with $z$. Finally, the $\mu / B$ ratios from the universal local pseudopotential EC(U) are always around 0.5 (Fig. 4). We have calculated the Voigt shear modulus $\mu$ by the method of Ref. 51. As a check on this method, we have reproduced the $\mathrm{EC}(\mathrm{I})$ values of $\mu$ obtained from the phonon frequencies of Ref. 4.

Cubic crystals have three independent elastic constants ${ }^{52}$ $C_{11}, C_{12}$, and $C_{44}$, or equivalently $B=\left(C_{11}+2 C_{12}\right) / 3, C^{\prime}$ $=\left(C_{11}-C_{12}\right) / 2$, and $\mu=\left(2 C^{\prime}+3 C_{44}\right) / 5$. $C^{\prime}$ and $C_{44}$ measure rigidity against tetragonal and angle-bending distortions of the unit cube, respectively. While $B$ and $\mu$ are essentially the same for the nearly-close-packed structures ${ }^{4,13} C^{\prime}$ can vary strongly between fcc and bcc structures. Our previous work ${ }^{4}$ with the individual pseudopotential $\mathrm{EC}(\mathrm{I})$ found $C^{\prime}$ $<0$ for all the real simple metals with $z \geqslant 2$ in the bcc structure, indicating a phonon soft-mode instability ${ }^{4,5}$ for this structure.

In Fig. 5 we present the interstitial electron number, as predicted by the individual pseudopotential EC(I), our former universal pseudopotential, ${ }^{3}$ and our present universal pseudopotential EC(U). For $z=1$ and 2 (but not 3 and 4), the former and present universal results are very similar.

Universality makes very simple predictions for the structures of solid alloys. For example, consider a binary alloy of metals $A$ and $B$, with concentrations $c$ and $(1-c)$, valences $z^{A}$ and $z^{B}$, and density parameters $r_{s}^{A}$ and $r_{s}^{B}$. The alloy valence is just

$$
z^{A B}=c z^{A}+(1-c) z^{B} .
$$

The alloy density parameter $r_{s}^{A B}$ is best found by minimizing the energy of the alloy, for example within the virtual-crystal approximation. ${ }^{2,53}$ While an accurate determination of $r_{s}^{A B}$ is needed to find the heat of formation, the simple Vegard rule ${ }^{2}$

$$
r_{s}^{A B}=c\left(\frac{z^{A}}{z^{A B}}\right)^{1 / 3} r_{s}^{A}+(1-c)\left(\frac{z^{B}}{z^{A B}}\right)^{1 / 3} r_{s}^{B}
$$

can suffice for the prediction of alloy crystal structure.

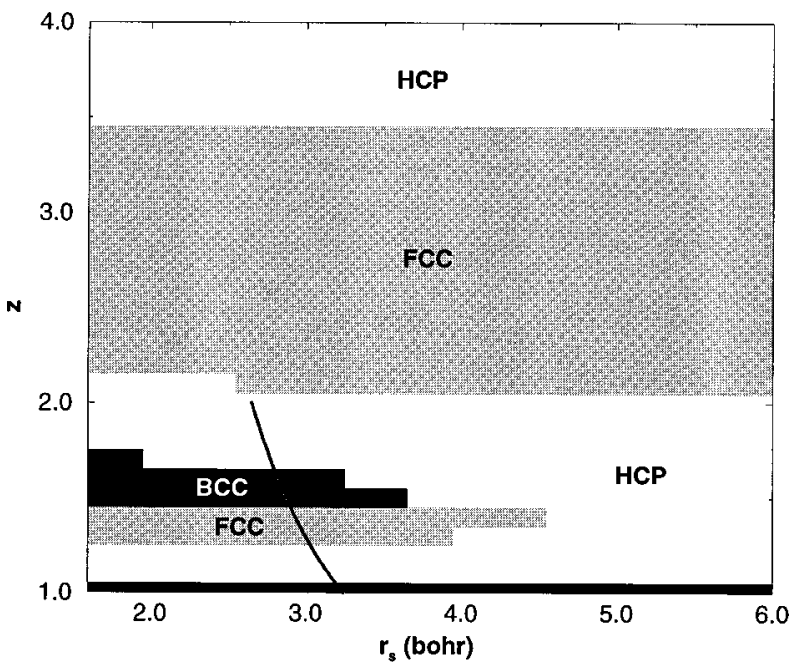

FIG. 8. Universal phase diagram at zero pressure. Shown is the structure of lowest energy for a given atomic volume. The line shows the $\mathrm{Li}_{1-c} \mathrm{Mg}_{c}$ phase transition.

To describe the stable structures of virtual crystals, the $\mathrm{EC}(\mathrm{U})$ parameters $(R, \alpha)$ were found in a regular mesh $\left(r_{s}, z\right)$. These pseudopotential parameters are available as a supplementary table. ${ }^{54}$ The phase diagram in the $\left(r_{s}, z\right)$ plane, for zero pressure, is shown in Fig. 8. There is a complicated pattern with the three compact structures interweaved, but $z$ is clearly the dominant factor determining the lattice. An example of alloy phase transitions is provided by $\mathrm{Li}_{1-c} \mathrm{Mg}_{c}$, with $c$ varying from 0 to 1 . The path for this transition can be seen in Fig. 8, and the structural energy differences are shown in Fig. 9. This alloy starts as bcc ( $c$ $=0, \mathrm{Li})$, and goes through several intermediate phases, arriving at hcp $(c=1, \mathrm{Mg})$. Figure 9 is very similar to Fig. 5 of Hafner, ${ }^{55}$ who used nonlocal pseudopotentials.

The Hume-Rothery rules ${ }^{53,56}$ for the structural phase transitions in alloys of noble metals with $s p$ metals predict the fcc- $\alpha$ phase to be stable up to electron number 1.38 , followed by the sequence of phases bcc- $\beta \rightarrow$ complex $\gamma$

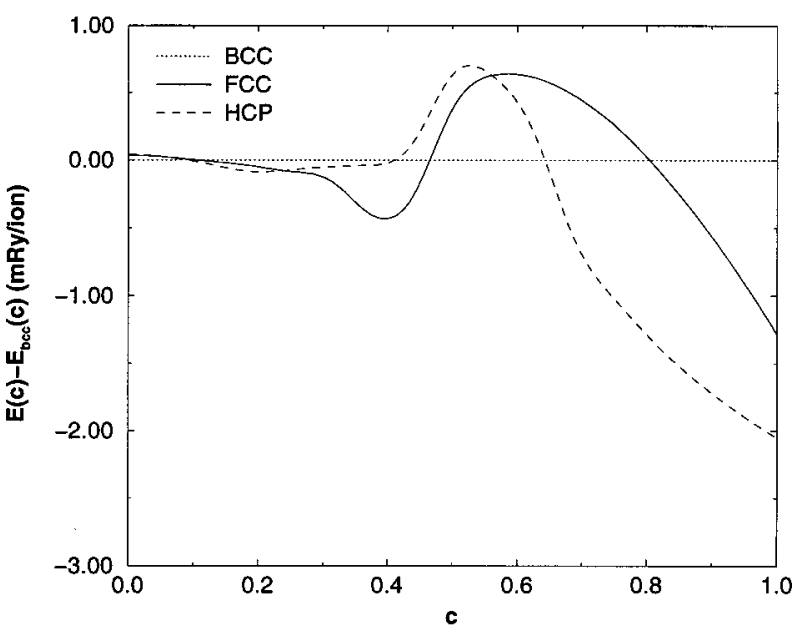

FIG. 9. Structural energy differences for the $\mathrm{Li}_{1-c} \mathrm{Mg}_{c}$ phase transition, from the universal local pseudopotential EC(U), for comparison with Fig. 5 of Ref. 55. Vegard's rule [Eq. (10)] has been employed here, but not in Ref. 55. 


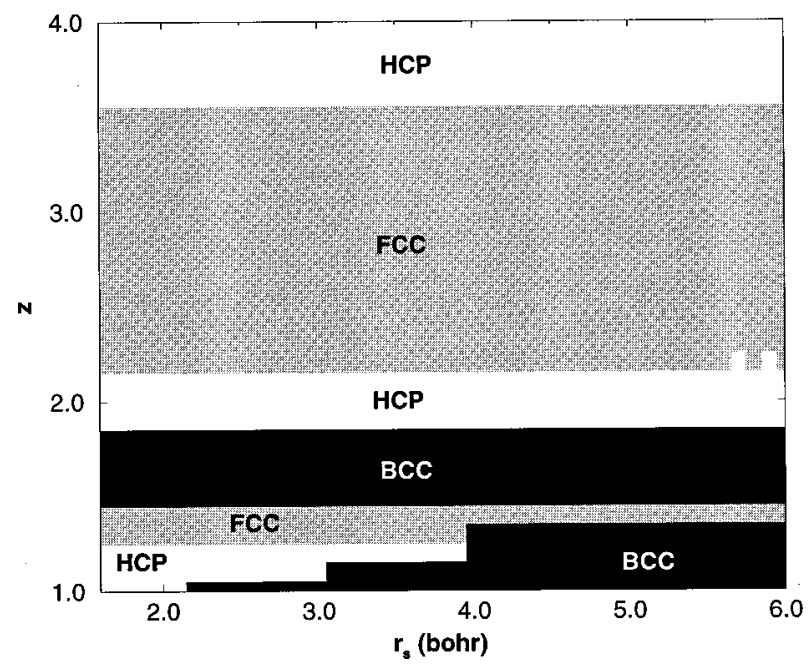

FIG. 10. Universal phase diagram at a compression ratio of $V / V_{0}=0.6$. According to the simplest realistic equation of state (Ref. 10), the pressure at this compression ratio is $P=B\left(0.31 B^{\prime}\right.$ $+0.18) . r_{s}$ is the density parameter for the uncompressed solid with $V / V_{0}=1$.

$\rightarrow$ hcp- $\epsilon$, stable at electron numbers 1.48, 1.62, and 1.75, respectively. From Fig. 8, for $r_{s}<4$, we can observe a similar pattern: fcc phase stable at $z=1.3-1.4$, bcc at $z=1.5-1.6$, and hcp at $z=1.7-2.0$.

In order to understand the mechanism of these transitions, we replace the Lindhard screening function $F\left[x=q /\left(2 k_{F}\right)\right]$ [Eq. (3.6) of Ref. 3] by a rational approximation ${ }^{57}$

$$
F^{\operatorname{approx}}(x)=\left(1+x^{2} / 3\right) /\left(1+2 x^{2} / 3+x^{4}\right) .
$$

Equation (11) matches the Lindhard function through order $x^{2}$ for small $x$, and through order $x^{-2}$ at large $x$. It matches the value (but not the infinite slope) of $F(x)$ at $x=1$. Under this replacement, the phase transitions described in the preceding paragraph all disappear. As argued in Ref. 58, the Hume-Rothery rules arise from the rapid variation of the Lindhard $F(x)$ around $x=1$. This Fourier-space argument can be complemented by one in real space. ${ }^{15,16}$

According to the Hume-Rothery size rule, ${ }^{2,56}$ substitutional alloys $A_{c} B_{1-c}$ tend to form only when $\left(z^{A}\right)^{1 / 3} r_{s}^{A}$ $\simeq\left(z^{B}\right)^{1 / 3} r_{s}^{B}$. Otherwise, they tend to phase separate. The element with the larger $r_{s}$ and thus the smaller surface energy is expected to segregate to the surface.

Figure 10 is the same as Fig. 8 but for a compressed volume ratio $V / V_{0}=0.6$. The most striking effect of pressure is the stabilization of the bcc structure for $z \leqslant 2$. The observed phase diagrams of the elements are summarized in Ref. 59. It would make a long story to discuss all the elements under pressure at zero temperature, so we focus on a few for which the local pseudopotential approximation is most appropriate: $\mathrm{Na}$ transforms to bcc under slight compression, and remains in that phase to $V / V_{0}=0.6$ or less. $\mathrm{Mg}$ remains hcp to $V / V_{0}=0.6$, then transforms to bcc. Al remains fcc to $V / V_{0}=0.6$ or less. Pb transforms to hcp under slight compression, and remains in that phase to $V / V_{0}=0.6$ or less. For these four elements, Fig. 10 is correct. We find the $\mathrm{Mg} \mathrm{hcp} \rightarrow$ bcc transition at $V / V_{0}=0.4-0.5$; the phonon soft mode of bcc Mg disappears for $V / V_{0} \leqslant 0.87^{4}$.

\section{CONCLUSIONS AND FUTURE DIRECTIONS}

The simplest realistic model for the simple metals is the stabilized jellium model with effective valence $z^{*}=1$, which has the single input parameter $r_{s}$ and correctly predicts surface properties and bulk moduli. The appropriate second level of theory is the universal local pseudopotential model we have presented here, which has two inputs $r_{s}$ and $z$, and correctly predicts additional properties including bulk binding energies, pressure derivatives of bulk moduli, shear moduli, and equilibrium crystal structures within secondorder perturbation theory.

Our universal local pseudopotential depends upon two parameters (other than $z$ ) which must be fixed by two realistic conditions. However, we have found that rather different two-parameter pseudopotentials (evanescent core and HeineAbarenkov) yield essentially the same results when fitted to the same set of conditions. We still prefer the evanescent core form, which is smoother in real space than the HeineAbarenkov form, and for which sums over reciprocal-lattice vectors converge faster; converged structural energy differences are found here by summing up to $G=8 k_{F}$, where $k_{F}$ $=(9 \pi / 4)^{1 / 3} / r_{s}$ is the Fermi wave vector.

The simplicity of our approach makes it well suited for the study of liquid metals, 4,60,61 expanded metals, 5,62 and solid or liquid alloys. For the solids at zero or high pressure, we have mapped the equilibrium crystal structures in the $r_{s}$ $-z$ plane. These predictions are rather realistic, except for the alkaline earths $(\mathrm{Ca}, \mathrm{Sr}, \mathrm{Ba})$ which have strongly nonlocal pseudopotentials.

Our pseudopotentials are constructed within and for a calculation of the energy to second order in perturbation theory. For use in nonperturbative calculations, the parameters should be reoptimized. ${ }^{4}$ This reoptimization represents a possible direction for future work, as it would allow us to expand Figs. 8 and 10 to include the diamond structure, an open crystal structure for which the pseudopotential is in no sense a weak perturbation. The simple $s p$-bonded elements include not only metals, but also semiconductors like $\mathrm{Si}$ which "prefer' the diamond structure. To find the $r_{s}$ which characterizes $z=4$ silicon, it is only necessary to perform an all-electron or nonlocal pseudopotential calculation for this element in a hypothetical fcc structure, as in Ref. $63\left(r_{s}\right.$ $=1.8$ bohr).

Finally, our work raises fundamental questions we can only partly answer: (1) Why must the form of a realistic pseudopotential depend upon at least two parameters other than the valence $z$ ? Chemical experience answers that a pseudopotential must minimally reproduce $z$ plus the ionization energy $I$ and the electron affinity $A$ of the free atom [or equivalently the electronegativity $(I+A) / 2$ and chemical hardness $I-A]$. (2) Why are $I$ and $A$ determined largely by $r_{s}$ and $z$ ? Our previous work ${ }^{19}$ on size effects in metal clusters provides an answer, if we regard an atom as a small cluster containing $z$ valence electrons. (3) Given that the bulk modulus $B$ is determined by $z$ and $r_{s}$, why is it so largely independent of $z$ for $z \geqslant 1$ ? While some trends in the simple metals can be explained ${ }^{64}$ by the structure of pseudopotential perturbation theory, this trend seems to arise from the internal structure of the atom. 


\section{ACKNOWLEDGMENTS}

This work was supported in part by the U.S. National Science Foundation under Grant Nos. DMR 95-21353 and DMR 98-10620, and by the Portuguese PRAXIS XXI pro- gram (Grant No. PRAXIS/2/2.1/FIS/473/94). Two of the authors (C.F. and J.P.P.) acknowledge the hospitality of the Institute for Nuclear Theory at the University of Washington, sponsored by the U.S. Department of Energy, where this work was completed.
${ }^{1}$ W. A. Harrison, Pseudopotentials in the Theory of Metals (Benjamin, New York, 1966).

${ }^{2}$ J. Hafner, From Hamiltonians to Phase Diagrams (SpringerVerlag, Berlin, 1987).

${ }^{3}$ C. Fiolhais, J. P. Perdew, S. Q. Armster, J. M. MacLaren, and M. Brajczewska, Phys. Rev. B 51, 14001 (1995); 53, 13 193(E) (1996).

${ }^{4}$ L. Pollack, J. P. Perdew, J. He, M. Marques, F. Nogueira, and C. Fiolhais, Phys. Rev. B 55, 15544 (1997).

${ }^{5}$ L. Pollack and J. P. Perdew, Int. J. Quantum Chem. 69, 359 (1998).

${ }^{6}$ F. Nogueira, C. Fiolhais, J. He, J. P. Perdew, and A. Rubio, J. Phys.: Condens. Matter 8, 287 (1996).

${ }^{7}$ J. P. Perdew, H. Q. Tran, and E. D. Smith, Phys. Rev. B 42, 11627 (1990).

${ }^{8}$ C. Fiolhais and J. P. Perdew, Phys. Rev. B 45, 6207 (1992).

${ }^{9}$ H. B. Shore and J. H. Rose, Phys. Rev. Lett. 66, 2519 (1991); J. M. Soler, ibid. 67, 3044 (1991).

${ }^{10}$ A. Alchagirov, J. P. Perdew, C. Fiolhais, J. C. Boettger, and R. C. Albers (unpublished).

${ }^{11}$ N. W. Ashcroft and D. C. Langreth, Phys. Rev. 155, 682 (1967).

${ }^{12}$ V. Heine and D. Weaire, in Solid State Physics, edited by H. Ehrenreich, F. Seitz, and D. Turnbull (Academic, New York, 1970), Vol. 24

${ }^{13}$ D. D. Ling and C. D. Gelatt, Phys. Rev. B 22, 557 (1980).

${ }^{14}$ J. P. Perdew and T. Datta, Phys. Status Solidi B 102, 283 (1980).

${ }^{15}$ J. Hafner and V. Heine, J. Phys. F 13, 2479 (1983).

${ }^{16}$ J. Hafner and V. Heine, J. Phys. F 16, 1429 (1986).

${ }^{17}$ K. A. Gschneidner, in Solid State Physics, edited by H. Ehrenreich, F. Seitz, and D. Turnbull (Academic, New York, 1966), Vol. 16. We have used this source to update the experimental values of the bulk moduli for $\mathrm{Ga}$ and $\mathrm{Sn}$.

${ }^{18}$ P. Vinet, J. H. Rose, J. Ferrante, and J. R. Smith, J. Phys.: Condens. Matter 1, 1941 (1989).

${ }^{19}$ M. Seidl, J. P. Perdew, M. Brajczewska, and C. Fiolhais, J. Chem. Phys. 108, 8182 (1998).

${ }^{20}$ R. M. Dreizler and E. K. U. Gross, Density Functional Theory (Springer-Verlag, Berlin, 1990).

${ }^{21}$ V. Heine and I. Abarenkov, Philos. Mag. 9, 451 (1964).

${ }^{22}$ I. Abarenkov and V. Heine, Philos. Mag. 12, 529 (1965).

${ }^{23}$ A. O. E. Animalu and V. Heine, Philos. Mag. 12, 1249 (1965).

${ }^{24}$ M. H. Cohen, J. Phys. Radium 23, 643 (1962).

${ }^{25}$ N. W. Ashcroft, Phys. Lett. 23, 48 (1966).

${ }^{26}$ R. W. Shaw, Phys. Rev. 174, 769 (1968).

${ }^{27}$ P. S. Ho, Phys. Rev. B 3, 4035 (1971).

${ }^{28}$ Z. D. Popovic, J. P. Carbotte, and G. R. Piercy, J. Phys. F 4, 351 (1974).

${ }^{29}$ R. N. Barnett, U. Landman, and C. L. Cleveland, Phys. Rev. B 27, 6534 (1983)

${ }^{30}$ A. G. Eguiluz, A. A. Maradudin, and R. F. Wallis, Phys. Rev. Lett. 60, 309 (1988)
${ }^{31}$ W.-D. Schöne, W. Ekardt, and J. M. Pacheco, Phys. Rev. B 50, 11079 (1994).

${ }^{32}$ F. Milstein and D. J. Rasky, Phys. Rev. B 54, 7016 (1996).

${ }^{33}$ F. Milstein, H. E. Fang, X.-Y. Gong, and D. J. Rasky, Solid State Commun. 99, 807 (1996).

${ }^{34}$ P. J. Feibelman, Phys. Rev. B 55, 8821 (1997).

${ }^{35}$ L. Goodwin, R. J. Needs, and V. Heine, J. Phys.: Condens. Matter 2, 351 (1990).

${ }^{36}$ I. J. Robertson, D. I. Thomson, V. Heine, and M. C. Payne, J. Phys.: Condens. Matter 6, 9963 (1994).

${ }^{37}$ N. Troullier and J. L. Martins, Solid State Commun. 74, 613 (1990).

${ }^{38}$ N. Troullier and J. L. Martins, Phys. Rev. B 43, 1993 (1991).

${ }^{39}$ D. R. Hamann, M. Schlüter, and C. Chiang, Phys. Rev. Lett. 43, 1494 (1979).

${ }^{40}$ G. B. Bachelet, D. R. Hamann, and M. Schlüter, Phys. Rev. B 26, 4199 (1982).

${ }^{41}$ D. R. Hamann, Phys. Rev. B 40, 2980 (1989).

${ }^{42}$ D. Vanderbilt, Phys. Rev. B 41, 7892 (1990).

${ }^{43}$ R. Berliner, H. G. Smith, J. R. D. Copley, and J. Trivisonno, Phys. Rev. B 46, 14436 (1992).

${ }^{44}$ C. Maier, O. Blaschko, and W. Pichl, Phys. Rev. B 55, 113 (1997).

${ }^{45}$ P. Staikov, A. Kara, and T. S. Rahman, J. Phys.: Condens. Matter 9, 2135 (1997).

${ }^{46}$ A. K. McMahan and J. A. Moriarty, Phys. Rev. B 27, 3235 (1983).

${ }^{47}$ M. M. Dacorogna and M. L. Cohen, Phys. Rev. B 34, 4996 (1986).

${ }^{48}$ J. C. Boettger and S. B. Trickey, Phys. Rev. B 53, 3007 (1996).

${ }^{49}$ M. L. Cohen, Phys. Rev. B 32, 7988 (1985).

${ }^{50}$ P. C. Kelires, Phys. Rev. B 55, 8784 (1997).

${ }^{51}$ T. Kraft, P. M. Marcus, M. Methfessel, and M. Scheffler, Phys. Rev. B 48, 5886 (1993).

${ }^{52}$ L. D. Landau and E. M. Lifschitz, Theory of Elasticity, 3rd ed. Course on Theoretical Physics Vol. 7 (Butterworth-Heinemann, New York, 1995).

${ }^{53}$ J. A. Alonso and N. H. March, Electrons in Metals and Alloys (Academic, London, 1989).

${ }^{54}$ See AIP Document No. E-PAPS:E-PRBMDO-59-106903 for a table with parameters $R$ and $\alpha$ of the $\mathrm{EC}(\mathrm{U})$ pseudopotential for several values of the average valence electron density $r_{s}$ and valence $z$. E-PAPS document files may be retrieved free of charge from our FTP server (http://www.aip.org/epaps/ epaps.html) or from ftp.aip.org in the directory /epaps/. For further information: e-mail: PAPS@aip.org or fax: 516-576-2223.

${ }^{55}$ J. Hafner, J. Phys. F 6, 1243 (1976).

${ }^{56} \mathrm{~W}$. Hume-Rothery, R. E. Smallman, and C. W. Haworth, The Structure of Metals and Alloys, 5th ed. (Institute of Metals, London, 1969).

${ }^{57}$ J. P. Perdew, Ph.D. thesis, Cornell University, 1971.

${ }^{58}$ D. Stroud and N. W. Ashcroft, J. Phys. F 1, 113 (1971). 
${ }^{59}$ D. A. Young, Phase Diagrams of the Elements (University of California Press, Los Angeles, 1991).

${ }^{60}$ M. Boulahbak, N. Jakse, J.-F. Wax, and J.-L. Bretonnet, J. Chem. Phys. 108, 2111 (1998).

${ }^{61}$ D. Chekmarev, M. Zhao, and S. A. Rice, J. Chem. Phys. 109, 768 (1998).
${ }^{62}$ W. C. Pilgrim, M. Ross, L. H. Yang, and F. Hensel, Phys. Rev. Lett. 78, 3685 (1997).

${ }^{63}$ M. T. Yin and M. L. Cohen, Phys. Rev. B 26, 5668 (1982).

${ }^{64}$ M. H. Cohen, in Alloying and Effects in Concentrated Solid Solutions, edited by T. B. Massalski (Gordon and Breach, New York, 1965), p. 1. 